\title{
BIAXIAL LOADING OF A PLATE CONTAINING A HOLE AND TWO CO-AXIAL THROUGH CRACKS
}

\author{
Heorgij SULYM*, Viktor OPANASOVYCH*, Mykola SLOBODIAN**, Yevhen YAREMA** \\ *Faculty of Mechanical Engineering, Bialystok University of Technology, ul. Wiejska 45C, 15-351 Bialystok, Poland \\ ${ }^{* *}$ Faculty of Mechanics and Mathematics, Department of Mechanics, \\ Ivan Franko National University of Lviv, Universytetska Str., 1, 79000 Lviv, Ukraine
}

sulym@pb.edu.pl, kafmech@franko.lviv.ua, slobkolia@gmail.com, evhenbro@gmail.com

received 21 April 2017, revised 24 September 2018, accepted 27 September 2018

\begin{abstract}
The paper presents the solution linear elasticity problem for an isotropic plate weakened by a hole and two co-axial cracks. The plate is exerted by uniform traction at infinity. The corresponding 2D problem is solved by the method of Kolosova-Muskhelishvili complex potentials. The method implies reduction of the problem to simultaneous singular integral equations (SIE) for the functions defined the region of the cracks and hole. For particular case the solution the SIE is obtained analytically in a closed form. A thorough analysis of the stress intensity factors (SIF) is carried out for various cases of the hole shape: penny-shaped, elliptical and rectangular.
\end{abstract}

Key words: Isotropic Plate, Co-Axial Cracks, Hole, Traction, Complex Potential, Stress Intensity Factors

\section{INTRODUCTION}

Thin walled elements, including thin plates, are used as part of modern machines and mechanisms as structural elements of buildings and constructions. By design they contain holes, while in the course of exploitation the small defects, like cracks, can originate and spread. Finally, these defects lead to the fracture of the element. The steady of the stress-strain state of plates containing cracks has been receiving considerable attention of many autors: Kaloerov et al. (2013), Kaminskii (1982), Kosmodamianskiy (1975), Kyt et al. (1983), Murakami (1990), Opanasovych et al. (2008), Panasyuk et al. (1976), Perez (2016), Savruk et al. (1981, 1988, 1989), Staschuk (1993), Sulym (2007).

The 2D plane crack problems are solved by using complex potentials, which leads to SIE for the functions defined at the cracks and at the contours of the holes: Chau et al. (1999), Chen et al. (2005, 2008, 2010, 2011, 2012), Hajimohamadi et al. (2018), Kratochvil et al. (2011), Kuliyev (2010), Lu et al. (2017), Maksymovych et al. (2017), Mogilevskaya et al. (2009), Pen et al. (2013), Savruk et al. (2007), Theocaris et al. (1989), Tsamasphyros et al. (2013), Wang H. et al. (2012), Wang J. et al. (2003), Wang X.-F. et al. (2000), Zemlyanova (2007), Zeng et al. (2018).

In the paper by Shao-Tzu et al. (2017) a new method has been developed recently. The authors of the paper: Felger et al. (2017), Gong (1994), Kushch et al. (2005), Liu et al. (2014) combined the complex potentials method with the superposition principle.

In the paper we utilize a commonly accepted approach of complex potentials to the problem for an isotropic plate containing a hole and two cracks.

\section{PROBLEM STATEMENT}

Let us consider a plane problem of linear elasticity for an isotropic plate containing a hole with a smooth contour and two co- axial cracks $2 l_{1}$ and $2 l_{2}$ in length. The plate is extended by a tensile uniform load $(p, q)$ applied at infinity. The cracks edges and the hole are free of loads. Let us introduce the Cartesian coordinate system $O x y$ in such a way that its origin is put at the center of one of the cracks, and the $O x$ axis is aligned along the cracks. Denoting the distance between the cracks centers $x_{0}$, the coordinates of the center of the other crack are $\left(x_{0}, 0\right)$, and tips are $a_{2}$ and $b_{2}$. Additionally, for the sake of convenience we introduce the auxiliary coordinate system $O^{\prime} x^{\prime} y^{\prime}$ associated with the hole. The transformation between the two coordinate systems is defined by the translation vector $(\mathrm{c}, \mathrm{d})$ and the angle of rotation $\beta$. The diagram of the problem is shown in Fig. 1 where we use the following nomenclature: $L=\tilde{L}_{1} \cup \tilde{L}_{2}$ - union of the straight segments where the cracks are located, $L_{1}$ - the curved contour of the hole. Further signs "+" and "-" denote the threshold value of the corresponding quantities at $\mathrm{y} \rightarrow \pm 0$.

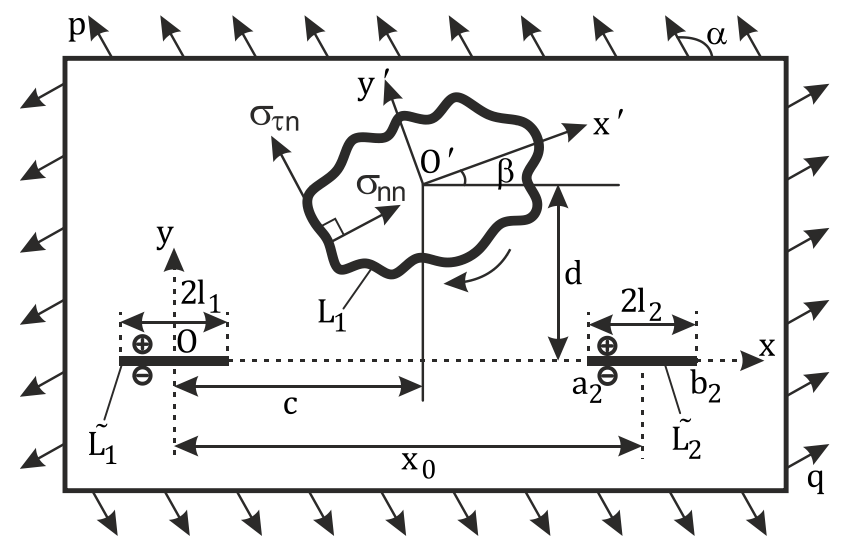

Fig. 1. Layout and loading of hole and cracks

The problem lies in finding the components of the stress tensor field $-\sigma_{y y}(x), \sigma_{x y}(x)$, and the components of external 
loading at the hole $-\sigma_{n n}$ and $\sigma_{\tau n}$, satisfying the following boundary conditions:

$\sigma_{y y}^{ \pm}(x)=0, \quad \sigma_{x y}^{ \pm}(x)=0, \quad x \in L$,

$\sigma_{n n}(t)=0, \quad \sigma_{\tau n}(t)=0, \quad t \in L_{1}$,

where the numbers with icons "+" and "-" are accordingly associated with the upper and lower edge of the crack.

\section{SOLUTION THE PROBLEM}

Following the well established approach, the stated 2D plane problem of elasticity can be conveniently solved by using the complex potentials of Kolosova-Mushelishvili: $\Phi(z), \Psi(z)$, $z=x+i y,(i=\sqrt{-1})$ (Mushelishvili, 1966). The components of the stress vector at the cracks edges $-L$ and at the contour of the hole $-L_{1}$ are given

$\sigma_{y y}-i \sigma_{x y}=\Phi(z)+\overline{\Phi(z)}+z \overline{\Phi^{\prime}(z)}+\overline{\Psi(z)}$,

$\sigma_{n n}+i \sigma_{\tau n}=\Phi(t)+\overline{\Phi(t)}+\frac{d \bar{t}}{d t}\left[t \overline{\Phi^{\prime}(t)}+\overline{\Psi(t)}\right]$

$t \in L_{1}$,

$\Phi(z)=\Gamma+\Phi_{1}(z)+\Phi_{2}(z)$,

$\Psi(z)=\Gamma^{\prime}+\Psi_{1}(z)+\Psi_{2}(z)$.

Savruk (1981) suggested representing $\Phi_{1}(z)$ and $\Psi_{1}(z)$ as $\Phi_{1}(z)=\frac{1}{2 \pi i} \int_{L_{1}} \frac{g_{1}(u) d u}{u-z}$,

$\Psi_{1}(z)=-\frac{1}{2 \pi i} \int_{L_{1}}\left[\frac{\overline{g_{1}(u)} d \bar{u}}{u-z}+\frac{\bar{u} g_{1}(u) d u}{(u-z)^{2}}\right]$

$g_{1}(u)$ - unknown function, $\Phi_{2}(z)$ i $\Psi_{2}(z)$ - holomorphic functions outside the cracks,

$\Gamma=\frac{1}{4}(p+q), \quad \Gamma^{\prime}=-\frac{1}{2}(p-q) e^{-2 i \alpha}$.

Function $g_{1}(u)$ defined at the contour of the hole satisfy the additional condition:

$\int_{L_{1}} g_{1}(u) d u=0$,

meaning that the main vector of the forces applied to the hole is equal to zero.

Let us introduce into the consideration function $\Omega_{2}(\mathrm{z})$ by the formula

$\Omega_{2}(z)=\overline{\Phi_{2}}(z)+z \overline{\Phi_{2}^{\prime}}(z)+\overline{\Psi_{2}}(z)$,

where $\overline{\Phi_{2}}(z)=\overline{\Phi_{2}(\bar{z})}$. form:

Taking into account (5) and (8), the relationship (3) takes the

$\sigma_{y y}-i \sigma_{x y}=\Phi_{2}(z)+\Omega_{2}(\bar{z})+(z-\bar{z}) \overline{\Phi_{2}^{\prime}(z)}+\Phi_{1}(z)+$

$+\overline{\Phi_{1}(z)}+z \overline{\Phi_{1}^{\prime}(z)}+\overline{\Psi_{1}(z)}+2 \Gamma+\bar{\Gamma}^{\prime}$.

Satisfying the boundary conditions (1), we finally got two conjugate problems for two functions $\Phi_{2}(z)$ and $\Omega_{2}(z)$ :

$\left(\Phi_{2}(x)-\Omega_{2}(x)\right)^{+}-\left(\Phi_{2}(x)-\Omega_{2}(x)\right)^{-}=0, x \in L$,

$\left(\Phi_{2}(x)-\Omega_{2}(x)\right)^{+}+\left(\Phi_{2}(x)-\Omega_{2}(x)\right)^{-}=0, x \in L$
The solution problems $(10,11)$ is given

$\Phi_{2}(z)=\Omega_{2}(z)$,

$\Phi_{2}(z)=\frac{1}{2 \pi i X(z)} \int_{L} \frac{f(x) X^{+}(x) d x}{x-z}+\frac{C_{0}}{X(z)^{\prime}}$

where:

$$
\begin{aligned}
& X(z)=\sqrt{\left(z^{2}-l_{1}^{2}\right)\left(z-a_{2}\right)\left(z-b_{2}\right)}, \\
& X^{+}(x)=\left\{\begin{array}{l}
-i \sqrt{\left(l_{1}^{2}-x^{2}\right)\left(-x+a_{2}\right)\left(-x+b_{2}\right)}, \quad x \in L_{1}, \\
i \sqrt{\left(x^{2}-l_{1}^{2}\right)\left(x-a_{2}\right)\left(x-b_{2}\right)}, \quad x \in L_{2}
\end{array}\right. \\
& f(x)=-\frac{1}{2 \pi i} \int_{L_{1}}\left\{g_{1}(u)\left[\frac{1}{u-x}+\frac{1}{\bar{u}-x}\right] d u-\right. \\
& \left.-\overline{g_{1}(u)}\left[\frac{1}{\bar{u}-x}+\frac{x}{(\bar{u}-x)^{2}}+\frac{u}{(\bar{u}-x)^{2}}\right] d \bar{u}\right\}-2 \Gamma-\bar{\Gamma}^{\prime},
\end{aligned}
$$

an unknown constant to be determined from the condition

$\int_{a_{2}}^{b_{2}}\left[\Phi_{2}^{+}(x)-\Phi_{2}^{-}(x)\right] d x=0$.

Substituting (13) in (15), we have:

$C_{0}=A+\int_{L_{1}}\left[g_{1}(u) B_{1}(u) d u+\overline{g_{1}(u)} B_{2}(u) d \bar{u}\right]$

where:

$$
\begin{aligned}
& A=\left(\Gamma+\frac{1}{2} \bar{\Gamma}^{\prime}\right) \sum_{m=1}^{N}\left(A _ { m } \left(x_{0}\left(l_{2} \tau_{m}+x_{0}\right)-\left(l_{2} \tau_{m}+x_{0}\right)^{2}+\right.\right. \\
& \left.\left.+\frac{l_{1}^{2}+l_{2}^{2}}{2}\right)\right) / \tilde{C}, \quad A_{m}=\frac{\pi}{N \sqrt{\left(\left(l_{2} \tau_{m}+x_{0}\right)^{2}-l_{1}^{2}\right)}}, \\
& \tilde{C}=\sum_{m=1}^{N} A_{m}, \\
& B_{1}(u)=-\frac{1}{2 \pi i} \sum_{m=1}^{N}\left(A_{m} \operatorname{Re}\left[\frac{X(u)}{u-l_{2} \tau_{m}-x_{0}}+u\right]\right) / \tilde{C}, \\
& B_{2}(u)=\frac{1}{4 \pi i} \sum_{m=1}^{N}\left(A _ { m } \left[\frac { u - \overline { u } } { \overline { u } - l _ { 2 } \tau _ { m } - x _ { 0 } } \left(y(\bar{u})+l_{2} \tau_{m}+\right.\right.\right. \\
& \left.\left.\left.+x_{0}-\bar{u}-\frac{m_{(u)}}{\bar{u}-l_{2} \tau_{m}-x_{0}}\right)\right]\right) / \tilde{C}, \tau_{m}=\cos \left(\frac{(2 m-1) \pi}{2 N}\right), \\
& y(z)=\frac{4 z^{3}-\left(3 z^{2}-l_{1}^{2}\right) 2 x_{0}+2 z\left(a_{2} b_{2}-l_{1}^{2}\right)}{2 X(z)} .
\end{aligned}
$$

Dependences (Mushelishvili, 1966)

$\int_{L} \frac{X^{+}(x) d x}{x-z}=\pi i\left(X(z)-z^{2}+z x_{0}+\frac{l_{1}^{2}+l_{2}^{2}}{2}\right)$,

$\int_{L} \frac{X^{+}(x) d x}{(x-z)^{2}}=\pi i\left(x_{0}-2 z+y(z)\right)$,

considering (17), function $\Phi_{2}(\mathrm{z})$ (13) takes the form:

$\Phi_{2}(z)=\int_{L_{1}}\left[g_{1}(u) R_{1}(u, z) d u+\overline{g_{1}(u)} R_{2}(u, z) d \bar{u}\right]+$
$+P(z)+\frac{C_{0}}{X(z)}$,

where

$P(z)=-\left(\Gamma+\frac{1}{2} \overline{\Gamma^{\prime}}\right)\left(1-\frac{1}{X(z)}\left(z^{2}-z x_{0}-\frac{l_{1}^{2}+l_{2}^{2}}{2}\right)\right)$, 
$R_{1}(u, z)=-\frac{1}{4 \pi i}\left[\frac{X(z)-\overline{X(u)}}{\bar{u}-z}+\frac{X(z)-X(u)}{u-z}+u+\bar{u}\right]$,

$R_{2}(u, z)=-\frac{1}{4 \pi i}\left[\frac{u-\bar{u}}{\bar{u}-z}\left(y(\bar{u})+\frac{X(z)-\overline{X(u)}}{\bar{u}-z}+z-\bar{u}\right)\right]$.

After taking into consideration (5), (8) and (12), function (4) can be represented as:

$$
\begin{aligned}
& \frac{d \bar{t}}{d t}\left(\Phi_{2}(\bar{t})+\bar{\Gamma}^{\prime}\right)+\overline{\Phi_{1}(t)}+\frac{d \bar{t}}{d t}\left[t \overline{\Phi_{1}^{\prime}(t)}+\overline{\Psi_{1}(t)}\right]+\Phi_{1}(t)+(19) \\
& +2 \Gamma+\Phi_{2}(t)+\left(1+\frac{d \bar{t}}{d t}\right) \overline{\Phi_{2}(t)}+(t-\bar{t}) \frac{d \bar{t}}{d t} \overline{\Phi_{2}^{\prime}(t)}=0, \quad t \in L_{1}
\end{aligned}
$$

Taking into account (6) and (18), formula (19) yields a singular integral equation for function $g_{1}(u)$ :

$$
\begin{aligned}
& \int_{L_{1}}\left[g_{1}(u) K(u, t) d u+\overline{g_{1}(u)} M(u, t) d \bar{u}\right]=\rho(t), \\
& t \in L_{1},
\end{aligned}
$$

where:

$$
\begin{aligned}
& K(u, t)=\frac{1}{2 \pi i}\left[\frac{1}{u-t}+\frac{d \bar{t}}{d t} \frac{1}{\bar{u}-\bar{t}}\right]+R_{1}(u, t)+\frac{B_{1}(u)}{X(t)}+ \\
& +\frac{d \bar{t}}{d t}\left(R_{1}(u, \bar{t})+\frac{B_{1}(u)}{X(t)}\right)+\left(1-\frac{d \bar{t}}{d t}\right)\left(\overline{R_{2}(u, t)}+\frac{\overline{B_{2}(u)}}{\overline{X(t)}}\right)+ \\
& +(t-\bar{t}) \frac{d \bar{t}}{d t}\left(\overline{R_{2}^{\prime}(u, t)}-\frac{\overline{y(t)}}{\overline{X^{2}(t)}} \overline{B_{2}(u)}\right), \\
& M(u, t)=-\frac{1}{2 \pi i}\left[\frac{1}{\bar{u}-\bar{t}}+\frac{d \bar{t}}{d t} \frac{t-u}{(\bar{u}-\bar{t})^{2}}\right]+R_{2}(u, t)+\frac{B_{2}(u)}{X(t)}+ \\
& +\frac{d \bar{t}}{d t}\left(R_{2}(u, \bar{t})+\frac{B_{2}(u)}{\overline{X(t)}}\right)+\left(1-\frac{d \bar{t}}{d t}\right)\left(\overline{R_{1}(u, t)}+\frac{\overline{B_{1}(u)}}{\overline{X(t)}}\right)+ \\
& +(t-\bar{t}) \frac{d \bar{t}}{d t}\left(\overline{R_{1}^{\prime}(u, t)}-\frac{\overline{y(t)}}{\overline{X^{2}(t)}} \overline{B_{1}(u)}\right), \\
& \rho(t)=-2 \Gamma-\frac{d \bar{t}}{d t} \bar{\Gamma}^{\prime}-P(t)-\frac{A}{X(t)}-\frac{d \bar{t}}{d t}\left(P(\bar{t})+\frac{A}{\overline{X(t)}}\right)- \\
& -\left(1-\frac{d \bar{t}}{d t}\right)\left(\overline{P(t)}+\frac{\bar{A}}{\overline{X(t)}}\right)-(t-\bar{t}) \frac{d \bar{t}}{d t}\left(\overline{P^{\prime}(t)}-\frac{\overline{y(t)}}{\overline{X^{2}(t)}} \bar{A}\right) .
\end{aligned}
$$

To estimate the safe range of external loading, we can use one of the criteria of fracture which takes into account the behavior of the stress vector components in the vicinity of the defects expressed through the SIF (Savruk, 1988):

$$
\begin{aligned}
& k_{11}^{ \pm}-i k_{12}^{ \pm}=2 \lim _{x \rightarrow \pm l}\left[\sqrt{2 \pi|x \mp l|} \Phi_{2}(x)\right], \\
& k_{21}^{-}-i k_{22}^{-}=2 \lim _{x \rightarrow a_{2}}\left[\sqrt{2 \pi\left|x-a_{2}\right|} \Phi_{2}(x)\right],
\end{aligned}
$$

$k_{21}^{+}-i k_{22}^{+}=2 \lim _{x \rightarrow b_{2}}\left[\sqrt{2 \pi\left|x-b_{2}\right|} \Phi_{2}(x)\right]$.

For further consideration we assume that the contour of hole $L_{1}$ is given by function $t^{\prime}=x^{\prime}+i y^{\prime}=\omega(\theta)=\widetilde{\omega}\left(e^{i \theta}\right)$, $0<\theta \leq 2 \pi$, with the specific properties:

$t(\theta)=c+i d+e^{i \beta} \omega(\theta)$.

By virtue of $u(v)=c+i d+e^{i \beta} \omega(v)$ and (22), equation (20) and relationship (7) take the form:

$\int_{0}^{2 \pi}[\tilde{g}(v) \widetilde{K}(v, \theta) d u+\overline{\tilde{g}(v)} \widetilde{M}(\nu, \theta) d v]=\tilde{\rho}(\theta)$,
$0<\theta \leq 2 \pi$,

$\widetilde{K}(v, \theta)=K(u(v), t(\theta)), \quad \widetilde{M}(v, \theta)=M(u(v), t(\theta))$,

$\tilde{\rho}(\theta)=\rho(t(\theta)), \quad \tilde{g}(v)=g_{1}(u(v)) \omega^{\prime}(v)$,

$\int_{0}^{2 \pi} \tilde{g}(v) d v=0$.

For $(23,24)$ we will use the method of numerical integration of SIE (Panasyuk et al., 1976).

\section{NUMERICAL ANALYSIS}

Numerical analysis of the problem was conducted at $\alpha=$ $\pi / 2, \beta=0, l_{1}=l_{2}=1$, with the interaction of cracks with elliptical (Fig. 2) and rectangular (Fig. 3) holes at $d / l=0$, $x_{0}=2 c$, and a circular hole (Fig. 4) at $d / l=1.2, c / l=0$, these figures curves 1 were built at $\tilde{q}=q / p=0$, curves 2 at $\tilde{q}=0.5$, curves 3 at $\tilde{q}=1$, curves 4 at $\tilde{q}=2$. For the considered cases of elliptical and rectangular holes $k_{11}^{ \pm}=k_{21}^{\mp}$, $k_{12}^{ \pm}=k_{22}^{\mp}=0$.

In Fig. 2 and Fig. 3 the panel (a) corresponds to distant cracks, while the panel (b) corresponds to the case of close cracks, relatively to the distance to the hole.

In the case of an elliptical hole, function $\omega(v)$ has the form:

$\omega(v)=R\left(e^{i v}+m e^{-i v}\right)$,

where: $R=(a+b) / 2, m=(a-b) /(a+b)$, a and $\mathrm{b}$ are semi-axes of the ellipse.

Fig. 2 illustrates the SIF $K_{1}^{* \pm}=k_{11}^{ \pm} /(p \sqrt{\pi l})$ as a function of the ellipse semi-axis $b$, normalized by l, provided all other parameters remain constant. From the graphs one can see the local maximum of SIF, behavior indicates of the parameter $b / l$ potentially dangerous from point of view of structural integrity, since in the vicinity of the local maximum the cracks can start to spread. The graphs also reveal the dependence of SIF on the ratio between the components of the traction vector $q / p$. However, such dependence is minor for the case of rectangular hole (Fig. 3).

For a rectangular hole, the reflecting function is taken as (Kosmodamianskiy, 1975)

$\omega(v)=R\left(e^{i v}+\sum_{k=1}^{9} \frac{\tilde{C}_{2 k-1}}{e^{i v(2 k-1)}}\right)$,

where $R$ is a real number, $\tilde{C}_{2 k-1}$ are known constants. 


\section{sciendo}

Heorgij Sulym, Viktor Opanasovich, Mykola Slobodian, Yevhen Yarema

Biaxial Loading of a Plate Containing a Hole and Two Co-Axial Through Cracks
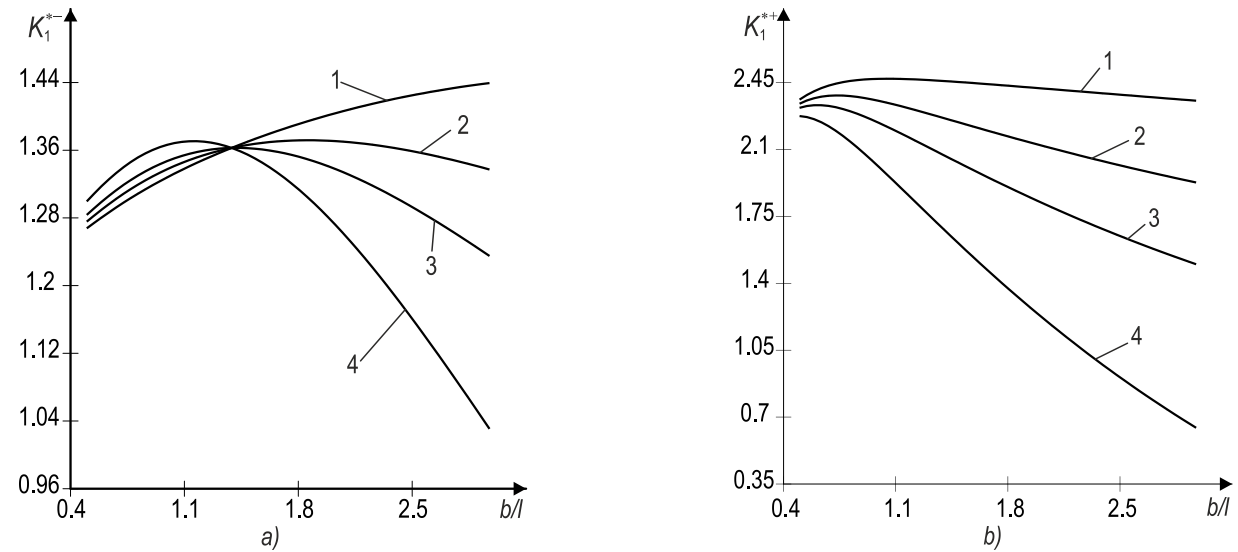

Fig. 2. Reduced intensity factors for an elliptical hole the ratio $b / l$ at different values $q / p$
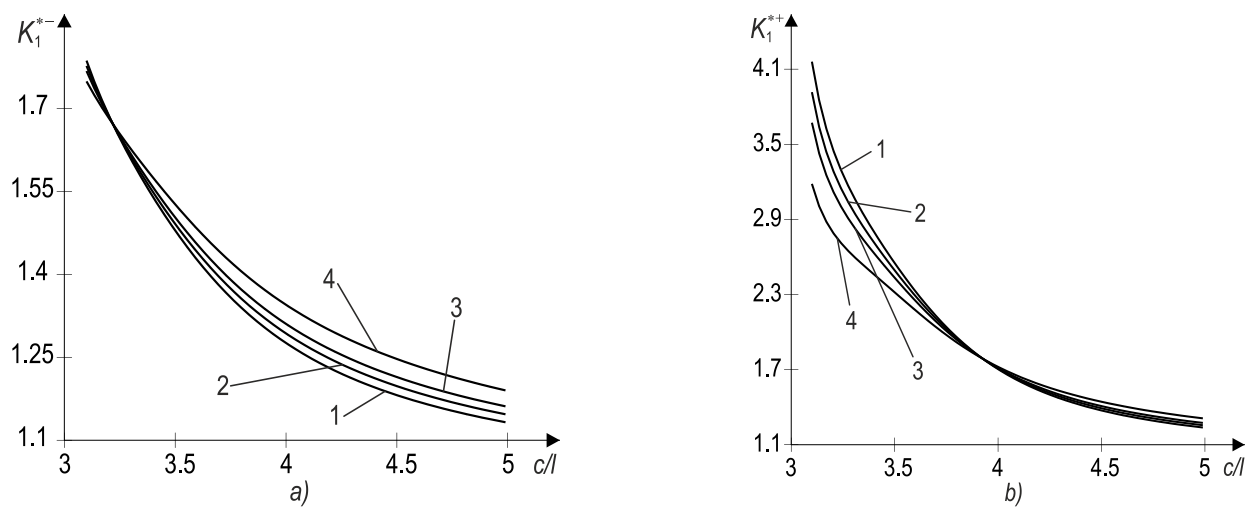

Fig. 3. Reduced intensity factors for a rectangular hole the ratio $c / l$ at different values $q / p$
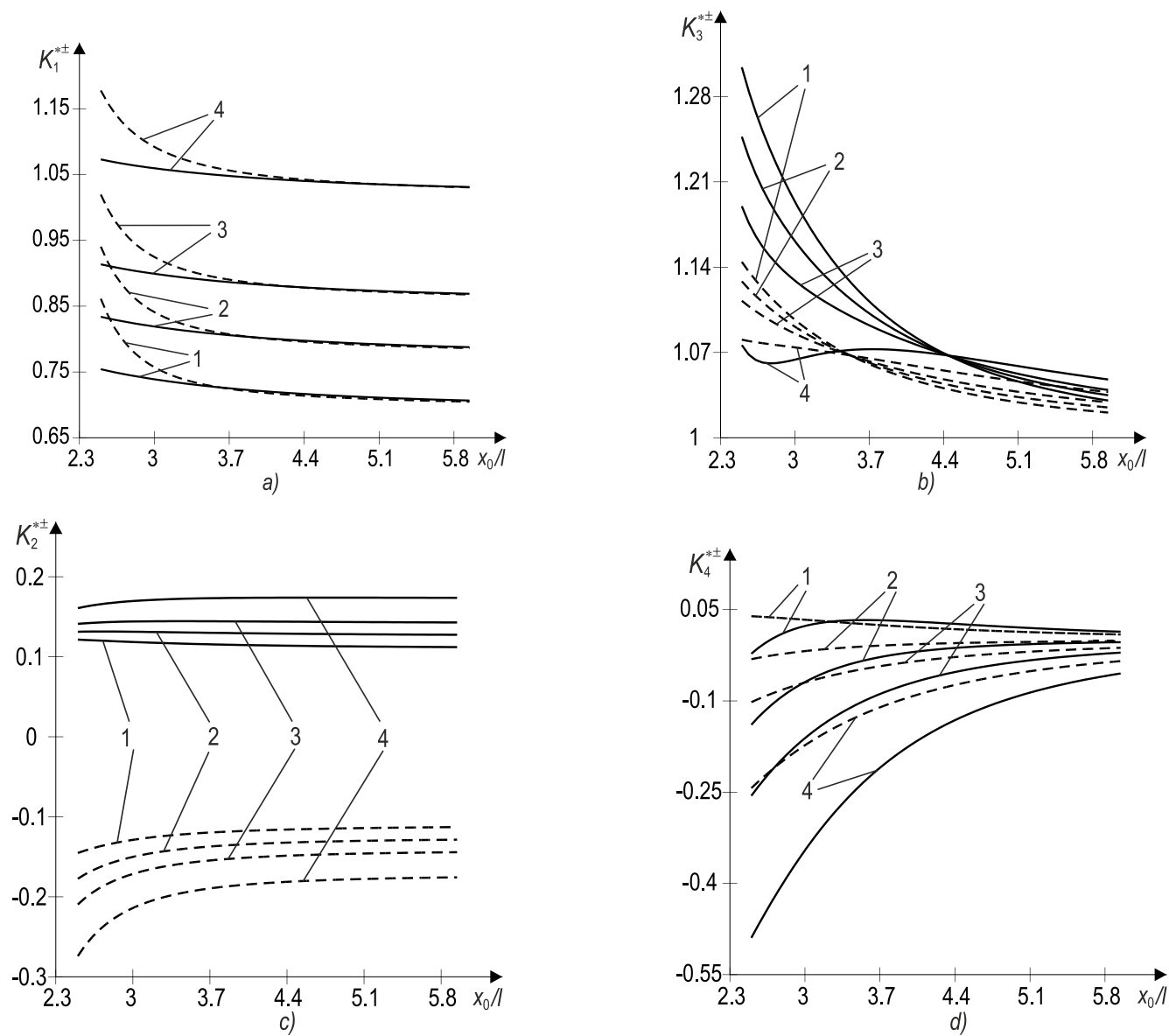

Fig. 4. Reduced intensity factors for a penny-shaped hole the ratio $x_{0} / l$ at different values $q / l$ 
Fig. 3 shows a graphical dependence of the consolidated SIF $K_{1}^{* \pm}=k_{11}^{ \pm} /(p \sqrt{\pi l})$ for a rectangular hole (with the ratio 2:1 between the sides, the bigger side is parallel to the cracks) on the relative distance $\mathrm{c} / \mathrm{l}$ between the center of the hole and the cracks centers.

The figure shows that the value of the SIF whether the tension is monoaxial or biaxial. However, when drawing the rectangular hole to the cracks, the SIF grow, and vice versa - go to the appropriate values for the isolated crack.

Fig. 4 illustrates the dependencies of SIFs $K_{j}^{* \pm}=$ $k_{1 j}^{ \pm} /(p \sqrt{\pi l}), \quad K_{2+j}^{* \pm}=k_{2 j}^{ \pm} /(p \sqrt{\pi l})(j=1,2)$ for a pennyshaped hole. The panels (a) and (c) correspond to the crack under the hole, while panels (b) and (d) correspond to the other one. The Fig. 4 illustrates the effect of interaction of two cracks the dependence of SIF's on the distance between the cracks $x_{0} / l$. The behavior of all functions $\operatorname{SIF}=\operatorname{SIF}\left(x_{0} / l\right)$ is monotonic; with increasing parameter $x_{0} / l$, the SIFs decrease approaching the corresponding limit values for the case of a single crack and the hole, know from (Kaloerov, 2013).

For the ratio $\mathrm{q} / \mathrm{p}$ increasing, the SIF the crack under the hole increases, while the SIF the other crack decreases.

\section{CONCLUSIONS}

The paper presents a solution 2D plane linear elasticity problem for the plate possessing a hole and two coaxial cracks in the field of uniform traction. By method of complex potentials the problem is reduced to a set of singular integral equations for functions defined at the hole contour. The numerical procedure (method of mechanical quadratures) was used to the approximate solution of the problem. The thorough analysis of the stress intensity factors SIF was carried out for particular cases of the hole shape and the location of the cracks relatively to the hole. Based on criteria of Fracture Mechanics, involving the SIFs, the analysis revealed the range of geometric parameters of the problem potentially dangerous from point of view of structural integrity of the plate.

\section{REFERENCES}

1. Chau K.T., Wang Y.B. (1999), A new boundary integral formulation for plane elastic bodies containing cracks and holes, International Journal of Solids and Structures, 36(14), 2041-2074.

2. Chen Y.Z. (2011), Solution of multiple crack problem in a finite plate using an alternating method based on two kinds of integral equation, Engineering Analysis with Boundary Elements, 35(10), 1109-1115.

3. Chen Y.Z. (2012), Boundary integral equation method for two dissimilar elastic inclusions in an infinite plate, Engineering Analysis with Boundary Elements, 36(2), 137-146.

4. Chen Y.Z., Lin X.Y. (2005), Periodic group crack problems in an infinite plate, International Journal of Solids and Structures, 42(9-10), 2837-2850.

5. Chen Y.Z., Lin X.Y. (2010), Solutions of the interior and exterior boundary value problems in plane elasticity by using dislocation distribution layer, International Journal of Solids and Structures, 47(34), 355-364.

6. Chen Y.Z., Wang Z.X., Lin X.Y. (2008), Crack front position and back position tichniques for evaluating the T-stress at crack tip using functions of a complex variable, Journal of Mechanics of Materials and Structure, 3(9), 1659-1673.
7. Felger J., Stein N., Becker W. (2017), Mixed-mode fracture in openhole composite plates of finite-width: An asymptotic coupled stress and energy approach, International Journal of Solids and Structures, 122-123, 14-24.

8. Gong S.X. (1994), Antiplane interaction of line crack with an arbitrarily located elliptical inclusion, Theoretical and Applied Fracture Mechanics, 20(3), 193-205.

9. Hajimohamadi M., Ghajar R. (2018), An analytical solution for the stress field and stress intensity factor in an infinite plane containing an elliptical hole with two unequal aligned cracks, Applied Mathematics and Mechanics, 39(8), 1103-1118.

10. Kaloerov S.A., Avdyushina Y.V., Myronenko A.B. (2013), Stress concentration in multiple isotropic plate, Naukova Dumka, Kyiv.

11. Kaminskii A.A. (1982), Brittle fracture near holes, Naukova Dumka, Kyiv.

12. Kosmodamianskiy A.S. (1975), The plane problem of the theory of elasticity for plates with holes, notches, projections, Vyshcha Shkola, Kyiv.

13. Kratochvil J., Becker W. (2011), Asymptotic analysis of stresses in an isotropic linear elastic plane or half-plane weakened by a finite number of holes, Archive of Applied Mechanics, 82(6), 743-754.

14. Kuliyev S.A. (2010), Uniform rotation of a polygonal plate weakened by two linear crack holes, Mechanics Research Communications, 37(2), 184-190.

15. Kushch V.I., Shmegera S.V., Buryachenko V.A. (2005), Interacting elliptic inclusions by the method of complex potentials, International Journal of Solids and Structures, 42(20), 5491-5512.

16. Kyt G.S., Krivcun M.G. (1983), Flat thermoelasticity problem for bodies with cracks, Naukova Dumka, Kyiv.

17. Liu S., Duan S. (2014), Analytical solutions of cracks emanating from an elliptic hole in an infinite plate under tension, Chinese Journal of Mechanical Engineering, 27(5), 1057-1063.

18. Lu A.-Z., Xu Z., Zhang N. (2017), Stress analytical solution for an infinite plane containing two holes, International Journal of Mechanical Sciences, 128-129, 224-234.

19. Maksymovych O., Illiushyn O. (2017), Stress calculation and optimization in composite plates with holes based on the modified integral equation method, Engineering Analysis with Boundary Elements, 83, 180-187.

20. Mogilevskaya S.G., Crouch S.L., Stolarski H.K. (2009), Interaction between a crack and a circular inhomogeneity with interface stiffness and tension, International Journal of Fracture, 159, 191.

21. Murakami Y. (1990), Handbook of stress intensity factor, Mir, Moscow.

22. Mushelishvili M.I. (1966), Some basic problems of the mathematical theory of elasticity, Nauka, Moscow.

23. Opanasovych V.K., Dorosh M. (2008), Combined bend with tension of plates weakened by two collinear cracks with contact of their faces, Bulletin of Lviv. Univ. Series meh.-mate, 68, 194-206.

24. Pan Z., Cheng Y., Liu J. (2013), Stress analysis of a finite plate with a rectangular hole subjected to uniaxial tension using modified stress functions, International Journal of Mechanical Sciences, 75, 265-277.

25. Panasyuk V.V., Savruk M.P., Datsishin A.P. (1976), Distribution of stresses around cracks in plates and shells, Naukova Dumka, Kyiv.

26. Perez N. (2016), Fracture mechanics, Springer International Publishing, Switzerland.

27. Savruk M, Kazberuk A. (2007), Stress intensity factors for diamondshaped hole in elastic plane under tension, Acta Mechanica et Automatica, 1(2), 45-48.

28. Savruk M.P, Osiv P.N, Prokopchuk I.V. (1989), Numerical analysis in plane problems of the crack theory, Naukova Dumka, Kyiv.

29. Savruk M.P. (1981), Two-dimensional problems of elasticity for bodies with cracks, Naukova Dumka, Kyiv.

30. Savruk M.P. (1988), The stress intensity factors in the bodies with cracks: a handbook. Fracture mechanics and strength of materials, Naukova Dumka, Kyiv. 
31. Shao-Tzu C.H., Li H. C.-C. (2017), Boundary-based finite element method for two-dimensional anisotropic elastic solids with multiple holes and cracks, Engineering Analysis with Boundary Elements, 79, 13-22.

32. Staschuk N.G. (1993), Problems of mechanics of elastic bodies with crack-like defect, Naukova Dumka, Kyiv.

33. Sulym G.T. (2007), Fundamentals of the mathematical theory of thermoelastic equilibrium deformed solids with thin inclusions, Research and Publishing Center of Shevchenko, Lviv.

34. Theocaris P.S., Petrou L. (1989), From the rectangular hole to the ideal crack, International Journal of Solids and Structures, 25(3), 213-233.

35. Tsamasphyros G.J., Theotokoglou E.E., Filopoulos S.P. (2013), Study and solution of BEM-singular integral equation method in the case of concentrated loads, International Journal of Solids and Structures, 50(10), 1634-1645.

36. Wang H., Qin Q.-H. (2012), A new special element for stress concentration analysis of a plate with elliptical holes, Acta Mechanica, 223(6), 1323-1340.
37. Wang J., Crouch S.L., Mogilevskaya S.G. (2003), A complex boundary integral method for multiple circular holes in an infinite plane, Engineering Analysis with Boundary Elements, 27(8), 789-802.

38. Wang X.-F., Hasebe N. (2000), Bending of a thin plate containing a rigid inclusion and a crack, Engineering Analysis with Boundary Elements, 24(2), 145-153.

39. Zemlyanova A. (2007), Singular integral equations for a patch repair problem, International Journal of Solids and Structures, 44, 6860-6877.

40. Zeng X.-T., Lu A.-Z., Zhang N. (2018), Analytical stress solution for an infinite plate containing two oval holes, European Journal of Mechanics - A/Solids, 67, 291-304. 